\title{
COMMENTS ON THE SEARCH FOR ELECTROSTATIC DISCHARGES ON MARS
}

\author{
Nilton O. RenNo ${ }^{1,2}$ AND Christopher S. RuF ${ }^{1,3}$ \\ ${ }^{1}$ Atmospheric, Oceanic and Space Sciences, University of Michigan, Ann Arbor, MI, USA; renno@ alum.mit.edu \\ ${ }^{2}$ Applied Physics Program, University of Michigan, Ann Arbor, MI, USA \\ ${ }^{3}$ Electrical Engineering and Computer Science, University of Michigan, Ann Arbor, MI, USA \\ Received 2012 March 16; accepted 2012 October 27; published 2012 November 28
}

\begin{abstract}
Ruf et al. used the Deep Space Network (DSN) to search for the emission of non-thermal radiation by martian dust storms, theoretically predicted by Renno et al. They detected the emission of non-thermal radiation that they were searching for, but were surprised that it contained spectral peaks suggesting modulation at various frequencies and their harmonics. Ruf et al. hypothesized that the emission of non-thermal radiation was caused by electric discharges in a deep convective dust storm, modulated by Schumann resonances (SRs). Anderson et al. used the Allen Telescope Array (ATA) to search for similar emissions. They stated that they found only radio frequency interference (RFI) during their search for non-thermal emission by martian dust storms and implicitly suggested that the signal detected by Ruf et al. was also RFI. However, their search was not conducted during the dust storm season when deep convective storms are most likely to occur. Here, we show that the ubiquitous dust devils and small-scale dust storms that were instead likely present during their observations are too shallow to excite SRs and produce the signals detected by Ruf et al. We also show that the spectral and temporal behavior of the signals detected by Anderson et al. corroborates the idea that they originated from man-made pulse-modulated telecommunication signals rather than martian electric discharges. In contrast, an identical presentation of the signals detected by Ruf et al. demonstrates that they do not resemble man-made signals. The presentation indicates that the DSN signals were consistent with modulation by martian SRs, as originally hypothesized by Ruf et al. We propose that a more comprehensive search for electrostatic discharges be conducted with either the ATA or DSN during a future martian dust storm season to test the hypothesis proposed by Ruf et al.
\end{abstract}

Key words: instrumentation: detectors - methods: data analysis - planets and satellites: individual (Mars) - radio continuum: planetary systems

Online-only material: color figures

\section{INTRODUCTION}

Recently, Anderson et al. (2011) used the Allen Telescope Array (ATA) to search for signals of electrostatic discharges on Mars. They did not find any evidence of emission of electrostatic discharges by Mars, but detected man-made signals and implicitly suggested that the signal detected by Ruf et al. (2009) was also man-made. Here we revisit the evidence presented by both Ruf et al. (2009) and Anderson et al. (2011) by adjusting both sets of measurements to a common spectral and temporal resolution. The common presentation confirms that Anderson et al. (2011) detected only man-made signals, whereas Ruf et al. (2009) detected signals likely originating in a deep convective martian dust storm, and not man-made signals.

The Anderson et al. (2011) statement that Ruf et al. (2009) detected non-thermal emission characterized by peaks in the power spectrum of the kurtosis at $10 \mathrm{~Hz}$ is incorrect. Ruf et al. (2009) found evidence for spectral peaks at several lower modes of the martian Schumann resonances (SRs) as well as at harmonics that provide evidence for a trigger mechanism. Ruf et al. (2009) show that the frequencies of these lower modes are within the range of values theoretically predicted for martian SRs. Tables 1 and 2 demonstrate that the differences between the theoretically predicted values and those observed by Ruf et al. (2009) are similar to differences between theory and observation in terrestrial SRs (Schumann 1952; Balser \& Wagner 1960; Morente et al. 2004).

The Anderson et al. (2011) statement that electrically active dust devils and small-scale dust storms are prevalent on Mars is correct but irrelevant; the electrical activity of these ubiquitous dust events is not intense enough to excite SRs. This is consistent with the fact that Ruf et al. (2009) spent $\sim 60 \mathrm{hr}$ searching for electric discharges on Mars and found evidence for it only during the measurement period ranging from 14:46 UTC to 22:46 UTC on 2006 June 8. The first detection of electric discharge by Ruf et al. (2009) occurred at 19:14 UTC and the signal was weak. Mars Express made a quick overpass nearby at 00:36 UTC, about $19 \mathrm{hr}$ before this first detection. Ruf et al. (2009) detected sporadic discharges in minute-long bursts, during a $2.5 \mathrm{hr}$ period. Nothing else similar to it occurred during 3 weeks of observations lasting 5-8 hr day ${ }^{-1}$, in spite of the Deep Space Network (DSN) field of view covering the entire martian disk. The ATA measurements reported in Anderson et al. (2011), on the other hand, contained recurring non-thermal signals throughout their $\sim 30 \mathrm{hr}$ of observations.

Anderson et al. (2011) also point to the null results of the Mars Express Advanced Radar for Subsurface and Ionospheric Sounding (MARSIS) in its search for electric activity (Gurnett et al. 2010), including on 2006 June 8 when Ruf et al. (2009) detected their signals, but hours before they detected any electric activity. However, besides lack of electric activity during the short MARSIS overpass near the storm area, this can also be explained by the different frequency ranges examined by MARSIS and by the DSN, in light of the theoretical predictions of the model by Renno et al. (2003).

MARSIS searched for signals between about $5 \mathrm{kHz}$ and $5 \mathrm{MHz}$, analogous to those generated by terrestrial lightning, while Ruf et al. (2009) used the DSN to search for the microwave 
Table 1

Terrestrial Schumann Resonances

\begin{tabular}{lccc}
\hline \hline SR Mode & Observed & Model & $f_{n}=f_{1} \sqrt{\frac{n(n+1)}{2}}$ \\
\hline$f_{l}(\mathrm{~Hz})$ & 7.8 & $8.3-10.6$ & 7.8 \\
$f_{2}(\mathrm{~Hz})$ & 14.1 & 15.8 & 13.5 \\
$f_{3}(\mathrm{~Hz})$ & 20.3 & 22.0 & 19.1 \\
$f_{4}(\mathrm{~Hz})$ & 26.4 & 29.4 & 24.7 \\
\hline
\end{tabular}

Notes. Observed values of the first four modes $\left(f_{1}, f_{2}, \ldots, f_{4}\right)$ of the terrestrial Schumann Resonances (SRs), values calculated by numerical models for a realistic cavity (model), and values calculated analytically for a perfect cavity (Schumann 1952; Balser \& Wagner 1960; Morente et al. 2004). The difference between the various values is as large as about $20 \%$

signals $(\sim 8.5 \mathrm{GHz})$ theoretically predicted to be produced by discharges between colliding dust particles.

The model proposed by Renno et al. (2003) predicts that the power and the spectral distribution of the emission of nonthermal radiation by dust storms are both functions of the particle radius. The model shows that the frequency of the emission of non-thermal radiation is $\omega \sim 0.1 / r^{2}$ (SI units), where $r$ is the radius of the particles. Since typical martian dust aerosols have diameters between 1 and $10 \mu \mathrm{m}$, they emit nonthermal radiation with peak power between 1 and $100 \mathrm{GHz}$. The emission of non-thermal radiation is expected to be vanishingly small at frequencies much lower than $1 \mathrm{GHz}$ because the number density of airborne particles with radius larger than $100 \mu \mathrm{m}$ is nearly zero. Particles this large are too heavy to be lifted by updrafts. Thus, the model proposed by Renno et al. (2003) predicts that no significant signal strength will be present at the frequencies between about $5 \mathrm{kHz}$ and $5 \mathrm{MHz}$ measured by MARSIS.

The statement that Gurnett et al. (2010) failed to detect impulsive radio signals over a period of five years that includes the 2006 June 8 storm is misleading for two reasons. Ruf et al. (2009) detected signals at frequencies of about $8.5 \mathrm{GHz}$, not around $5 \mathrm{MHz}$. In addition, since typical dust devils and dust storms produce a charge moment that is orders of magnitude smaller than that of the deep convective dust storm observed on 2006 June 8, they are unlikely to force SRs and produce the non-thermal radiation detected by Ruf et al. (2009), as explained below.

\section{FORCING OF SCHUMANN RESONANCES}

The forcing of SRs by electric discharges is proportional to the change in charge moment produced by them. The maximum charge moment of a dust devil or dust storm is $M_{\mathrm{Max}}=z A \varepsilon E_{\mathrm{Max}}$, where $z$ is the depth of the dust devil or dust storm, $A$ is the area covered by the dust plume, $\varepsilon \approx 8.85 \times 10^{-12} \mathrm{~F} \mathrm{~m}^{-1}$ is the electric permittivity of the martian air ( $\sim$ free space), and $E_{\mathrm{Max}}$ is the maximum electric field in the dust storm, taken as the nearly critical (close to the breakdown value) electric field amplitude $E_{\max } \sim 20 \mathrm{kV} \mathrm{m}^{-1}$. Ruf et al. (2009) estimated $M_{\text {Max }} \approx$ $10^{9} \mathrm{C} \mathrm{m}$ for the 2006 June 8 dust storm. For a typical dust devil, less than $1 \mathrm{~km}$ deep with dust cloud area smaller than $1 \mathrm{~km}^{2}$, we find $M_{\mathrm{Max}}<<10^{2} \mathrm{C} \mathrm{m}$. Ruf et al. (2009) calculate the maximum forcing of the SRs by assuming that the dust storm would be completely discharged during the minute long bursts seen in the kurtosis. In this case, the averaged rate of charge transfer squared would be $10^{16}(\mathrm{C} \mathrm{m})^{2} \mathrm{~s}^{-1}$ for the 2006 June 8 dust storm and less than $1(\mathrm{C} \mathrm{m})^{2} \mathrm{~s}^{-1}$ for typical dust devils. Thus, the forcing of SRs by a typical dust devil is at
Table 2

Martian Schumann Resonances

\begin{tabular}{lccc}
\hline \hline SR Mode & Observed & Model & $f_{n}=f_{1} \sqrt{\frac{n(n+1)}{2}}$ \\
\hline$f_{l}(\mathrm{~Hz})$ & 9.6 & $8.8-14.3$ & 9.6 \\
$f_{2}(\mathrm{~Hz})$ & 19.2 & $16.1-25.8$ & 16.6 \\
$f_{3}(\mathrm{~Hz})$ & 27.8 & $23.6-37.4$ & 23.5 \\
$f_{4}(\mathrm{~Hz})$ & 31.7 & $\ldots$ & 30.4 \\
\hline
\end{tabular}

Note. Same as Table 1, but for martian SRs. The difference between the various values is similar to that observed for terrestrial SRs

least $10^{16}$ times smaller than that of the 2006 June dust storm. Typical dust storms are shallow, no more than a few $\mathrm{km}$ deep and much less likely to produce charge separation as large as storms with rapid vertical development such as the 2006 June 8 dust storm; therefore, they produce a charge moment at least an order of magnitude smaller than the 2006 June 8 dust storm. For this reason, the forcing of SRs by typically shallow dust storm of similar area is at least $\sim 10^{2}$ times smaller that of the 2006 June 8 dust storm. Shallower dust storms of larger area are not uncommon, but it is unlikely that the entire dust storm would be electrically active. Convective dust storms of large vertical growth and capable of being strongly electrified, such as terrestrial thunderstorms and similar to the 2006 June storm, are more rare. Therefore, the 2006 June 8 convective dust storm is not at all the typical event suggested by Anderson et al. (2011).

\section{DATA ANALYSIS}

Here we show that the signals detected by the ATA resemble man-made pulse-modulated telecommunication signals. In contrast, the signals detected by the DSN during the 2006 June 8 deep convective dust storm do not resemble man-made signals.

The Anderson et al. (2011, p. 12) statement that "The frequency structure and timescales of the signals seen in the kurtstrum (i.e., spectral kurtosis) strongly resemble those detected by Ruf et al. (2009) during the 2006 June 8 large-scale dust storm event" is incorrect. The temporal and spectral dependencies of the signals are dissimilar in important ways that suggest very different sources for the two signals. The Anderson et al. (2011) observations were made with finer temporal and spectral resolution (1.25 $\mathrm{ms}$ and $0.102 \mathrm{MHz}$, respectively) than were those in Ruf et al. (2009), which were 4.2 ms and 2.5 MHz. The resolution of the Anderson et al. (2011) observations has been degraded by averaging to $3.75 \mathrm{~ms}$ and $2.46 \mathrm{MHz}$ in order to more directly compare them with those of Ruf et al. (2009). A 30 s time interval of both observations in each of eight contiguous spectral bands is shown in Figure 1 for periods when strong non-thermal signals are present. Two significant differences are apparent between the observations. The spectrum of the Ruf et al. (2009) observations extends over all eight sub-bands, whereas the Anderson et al. (2011) observations are limited to just sub-bands 2 and 3. Signals with sharply restricted spectral extent are a common characteristic of man-made telecommunications. The time dependencies of the two signals are also quite different. In the case of Ruf et al. (2009), the non-thermal events tend to persist for several seconds or more. The Anderson et al. (2011) non-thermal events, on the other hand, occur in brief bursts. This can more easily be seen in Figure 2, which zooms in on a shorter, $3 \mathrm{~s}$, subset of the data from Figure 1. The non-thermal events in the two cases have very different time dependencies. In the case of the Anderson et al. (2011) observations, there are clear intervals of time between the short non-thermal bursts in 

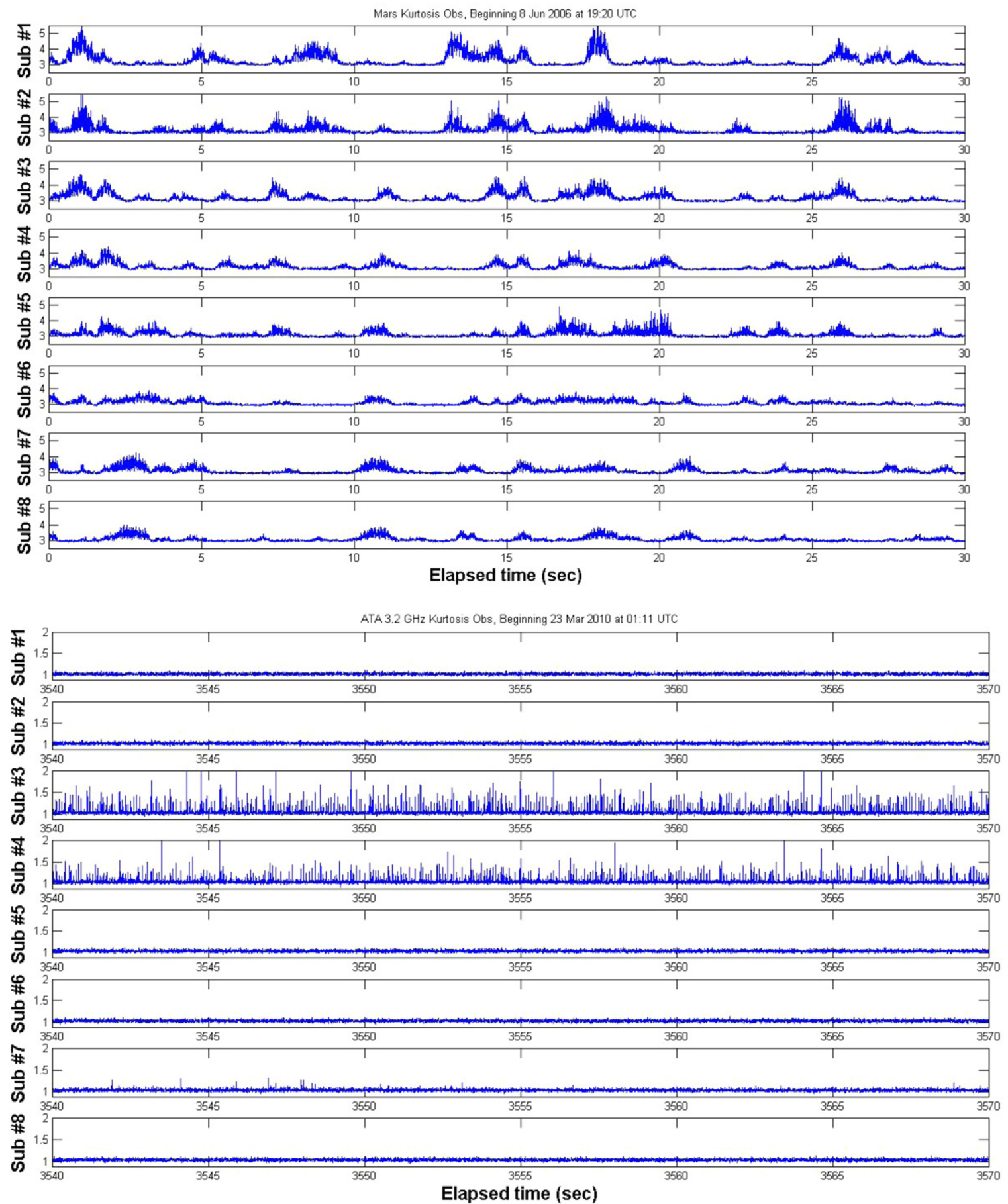

Figure 1. Comparison between Ruf et al. (2009) and Anderson et al. (2011) observations of non-thermal signals while viewing Mars. Top: observations of kurtosis at eight spectral bands $(8470-8472.5,8472.5-8475, \ldots, 8487.5-8490 \mathrm{MHz})$ on 2006 June 8 . Bottom: observations of spectral kurtosis at eight spectral bands (3190.1-3192.4 3192.5-3194.9, 3195-3197.3, 3197.4-3199.8, 3200.2-3202.6, 3202.7-3205.0, 3205.1-3207-5, 3207.6-3210.0) on 2010 March 23. Deviations from 3 (top) and 1 (bottom) indicate the presence of a signal with non-Gaussian amplitude distribution.

(A color version of this figure is available in the online journal.)

which no signal is present. This is also a common characteristic of man-made pulse-modulated telecommunication signals. The Ruf et al. (2009) observations, on the other hand, do not resemble any common type of telecommunication signal.

The Anderson et al. (2011, p. 10) statement that " $10 \mathrm{~Hz}$ variations in the kurtstrum are consistent with the expectations for electrostatic discharges as described by Ruf et al. (2009)" is not supported by a careful examination of the observations. In fact, the spectral composition of the two signals is quite different. Power spectra derived from $10 \mathrm{~s}$ segments of each of the two signals from Figure 1 are shown in Figure 3. The Ruf et al. (2009) spectrum contains frequency components at three SR frequencies $(9.6,27.8$, and $31.6 \mathrm{~Hz}$ ) and at a number of exact integer multiples of those frequencies (9.6 $\times 2, \times 4, \times 5, \times 7, \times 10 ; 27.8 \times 2, \times 4$; and $31.6 \times 3)$. As discussed by Ruf et al. (2009), the presence of these harmonics is evidence of a trigger mechanism by the SR modes. Apart from these frequency components, there is very little spectral content in the Ruf et al. (2009) signal. The power spectrum of the Anderson et al. (2011) signal shown in Figure 3, on the 

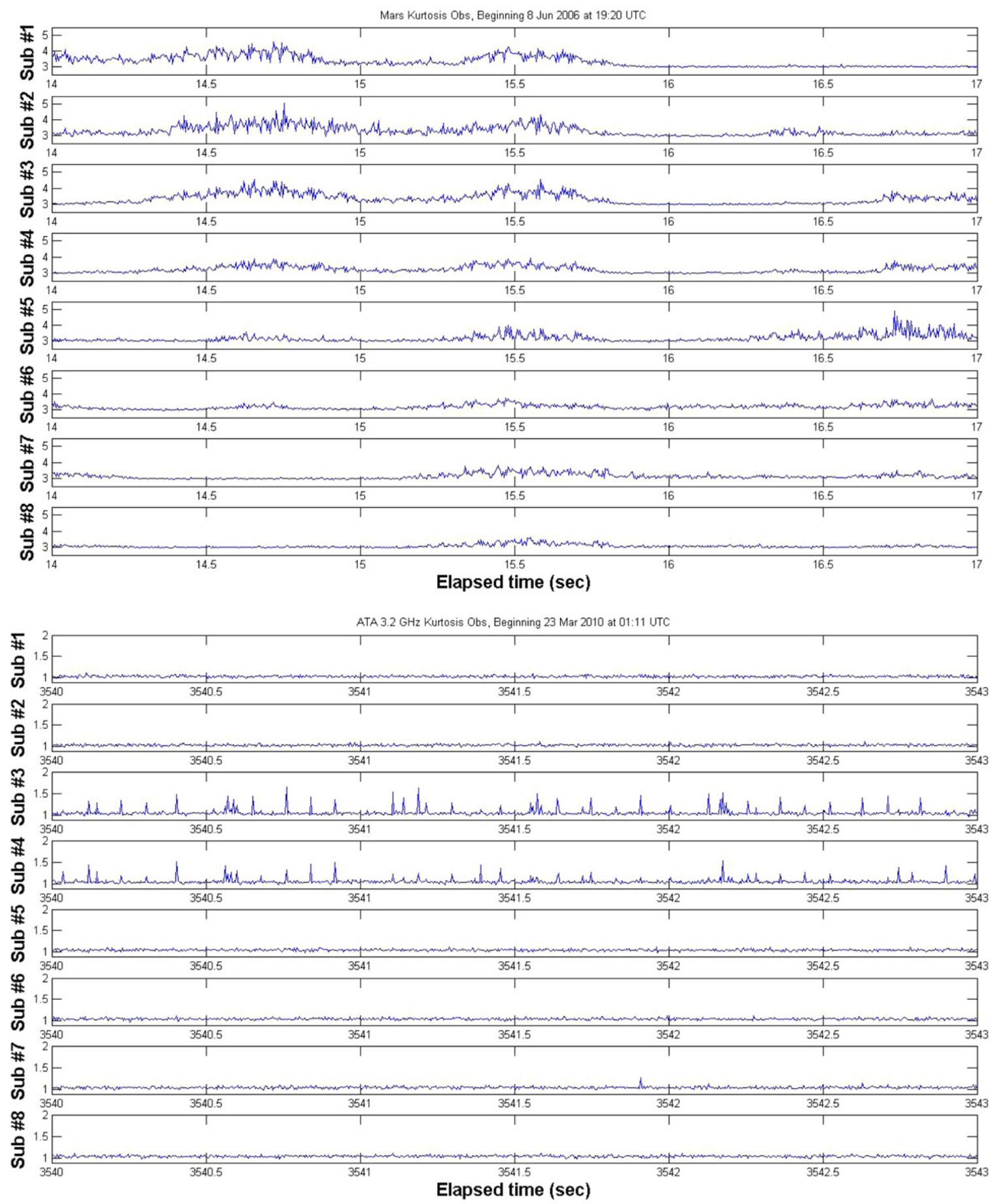

Figure 2. Same as Figure 1, but for a $3 \mathrm{~s}$ subset of the data. The temporal resolution in both cases is $4 \mathrm{~ms}$. The 2006 June 8 observations (top) contain non-thermal signals that persist for multiple seconds, whereas the 2010 March 23 observations (bottom) consistent of short, isolated bursts of signal.

(A color version of this figure is available in the online journal.)

other hand, contains one prominent spectral feature at $11.2 \mathrm{~Hz}$ plus a large number of additional spectral components. Notably, the spectrum does not appear to contain integer harmonics of the $11.2 \mathrm{~Hz}$ component or of any other of its larger spectral features. In fact, the Anderson et al. (2011) spectrum has the dense spectral distribution common to many spread spectrumtype communication protocols.

Anderson et al. (2011, p. 11) note that the non-thermal signals they presented were typical and occurred "on average one to two times per hour" during the entire $30 \mathrm{hr}$ of their observa- tions. This fact, plus the similarity between their observations' spectral and temporal features and those of common man-made telecommunication signals, suggests that the ATA observations at $3.2 \mathrm{GHz}$ were made in a highly radio frequency interference (RFI) contaminated environment. The non-thermal signals observed by Ruf et al. (2009) have several distinguishing differences and do not resemble typical RFI.

Table 1 shows observed values of the first four modes of the terrestrial SRs, values calculated by numerical models for a realistic cavity, and values calculated analytically for a perfect 

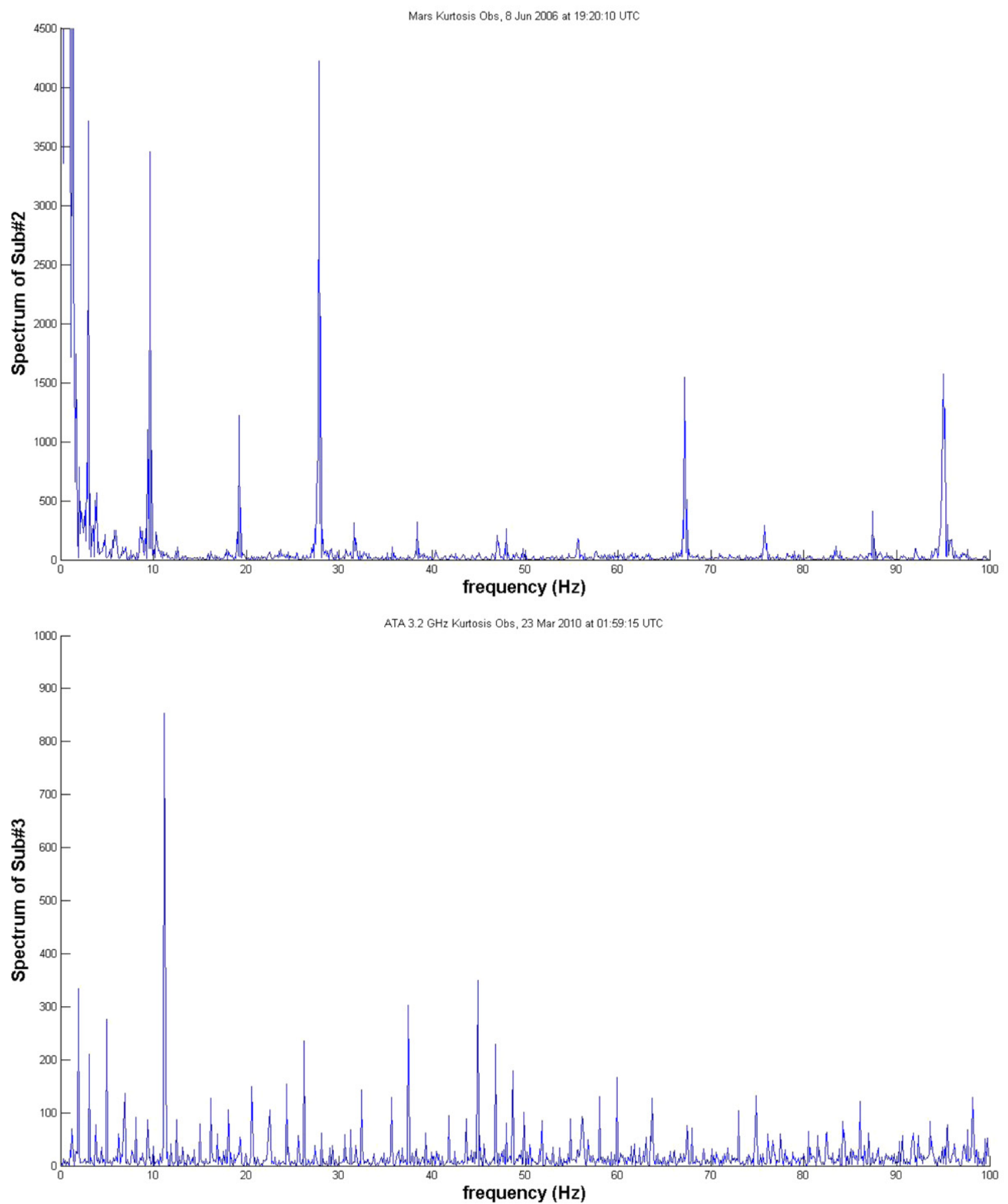

Figure 3. Comparison between power spectra derived from $10 \mathrm{~s}$ time records for Ruf et al. (2009) and Anderson et al. (2011) observations of non-thermal signals while viewing Mars. Top: observations of kurtosis at 8472.5-8475 MHz on 2006 June 8. Bottom: observations of spectral kurtosis at 3195-3197.3 on 2010 March 23. (A color version of this figure is available in the online journal.)

cavity (Schumann 1952; Balser \& Wagner 1960; Morente et al. 2004). The difference between the various values is as large as about $20 \%$. Table 2 shows similar analysis for Mars (Ruf et al. 2009), indicating that the differences between the various values are of the same order. However, the second mode of the martian SRs is an exact multiple of the first mode suggesting phase locking by the triggering mechanism postulated by Ruf et al. (2009). The result of this analysis is also consistent with the idea that Ruf et al. (2009) detected natural signals consistent with martian SR signatures, not RFI.

\section{CONCLUSIONS}

Detailed examination of the spectral and temporal behavior of the signals detected by Anderson et al. (2011) confirm that they probably originated from man-made pulse-modulated telecommunication signals rather than martian electric discharge. In contrast, an identical examination of the signals detected by Ruf et al. (2009) during the 2006 June 8 convective dust storm reveals features that are markedly different from common man-made signals. The signals detected by Ruf et al. (2009) were consistent with modulation by martian SRs, as originally 
hypothesized. The hypothesis proposed by Ruf et al. (2009) should be tested by measurements with either the ATA or the DSN during the martian dust storm season when deep convective dust storms are more likely to occur. In fact, the finer spectral and temporal resolutions provided by the ATA observing system make further observations by it an attractive opportunity to improve upon the earlier DSN results.

The authors thank NSF for financial support through award AGS 1119467 and the editor and reviewers for constructive comments and suggestions.

\section{REFERENCES}

Anderson, K. M., Siemion, A. P. V., Barott, W. C., et al. 2011, ApJ, 744, 15 Balser, M., \& Wagner, C. A. 1960, J. Research N.B.S., 64D, 415

Gurnett, D. A., Morgan, D. D., Granoth, L. J., et al. 2010, Geophys. Res. Lett., 37, L17802

Morente, J. A., Porti, J. A., Salinas, A., et al. 2004, J. Geophys. Res., 109,2309

Renno, N. O., Wong, A. S., Atreya, S. K., et al. 2003, Geophys. Res. Lett., 30, 2140

Ruf, C., Renno, N. O., Kok, J. F., et al. 2009, Geophys. Res. Lett., 36, L13202

Schumann, W. O. 1952, Z. Nat., A72, 250 